\title{
DISAPPEARANCE OF GLYCOPROTEIN IB FROM THE PLATELET SURFACE IN PERICARDIAL BLOOD DURING CARDIOPULMONARY BYPASS
}

Kyra N. Maquelin, MD ${ }^{\mathrm{a}}$ René J. Berckmans ${ }^{\mathrm{b}}$

Rienk Nieuwland, $\mathrm{PhD}^{\mathrm{b}}$

Marianne C. L. Schaap ${ }^{b}$

Klaas ten Have, MD ${ }^{\mathrm{a}}$

Leòn Eijsman, $\mathrm{MD}, \mathrm{PhD}^{\mathrm{a}}$

Augueste Sturk, $\mathrm{PhD}^{\mathrm{b}}$
Objectives: Several investigators have reported decreased expression of glycoprotein Ib on the platelet surface during coronary artery bypass grafting, but others could not confirm this finding. Because platelet glycoprotein Ib functions as an adhesion receptor for von Willebrand factor and other adhesive proteins, this decreased expression may explain excessive postoperative blood loss. In this study the expressions of glycoprotein Ib and other platelet activation markers were studied in the systemic and pericardial blood of seven patients undergoing coronary artery bypass grafting. Pericardial blood was recently shown to have high activation levels of fibrinolytic and coagulation pathways; we hypothesized that this local blood activation might be paralleled by extensive platelet activation and associated disappearance of glycoprotein Ib. Methods: Expression of platelet surface antigens was determined by whole-blood double-label flow cytometry. Results: Glycoprotein Ib expression in systemic blood decreased $10 \%(p=0.03)$ from preoperative levels at the start of cardiopulmonary bypass and $30 \%(p=0.04)$ before release of the aortic crossclamp. Expression in pericardial blood at these times decreased by $50 \%$ and $51 \%$, respectively $(p=0.003, p=0.009)$. No changes were observed in the expression of the platelet activation antigens CD62P (P-selectin, indicating platelet $\alpha$-granular release) and CD63 (indicating lysosomal release) or in binding of monoclonal antibody PAC-1 (detecting the fibrinogen-binding receptor conformation of the glycoprotein IIb-IIIa complex). Conclusion: Glycoprotein Ib disappeared from the platelet surface during bypass grafting, most notably in pericardial blood. No increased expression of CD62P, CD63, or PAC-1 was found, indicating the absence of general platelet activation. (J Thorac Cardiovasc Surg 1998;115:1160-5)
$\mathrm{D}$ uring cardiopulmonary bypass $(\mathrm{CPB})$ the patient's blood is in extensive contact with the artificial surface of the extracorporeal circuit. This contact results in activation of the factor XIIdependent contact system and thus both the coagulation cascade and the fibrinolytic system. ${ }^{1}$ Recent improvements in biocompatibility of the extracorpo-

From the Department of Cardiopulmonary Surgery, Onze Lieve Vrouwe Gasthuis, Amsterdam, ${ }^{\mathrm{a}}$ and the Department of Clinical Chemistry, Leiden University Medical Center, Leiden, ${ }^{\mathrm{b}}$ The Netherlands.

Received for publication July 22, 1997; revisions requested Sept. 2, 1997; revisions received Oct. 30, 1997; accepted for publication Nov. 14, 1997.

Address for reprints, K. N. Maquelin, MD, Onze Lieve Vrouwe Gasthuis, Department of Cardiopulmonary Surgery, P.O. Box 95500, 1090 HM Amsterdam, The Netherlands.

Copyright (C) 1998 by Mosby, Inc.

$0022-5223 / 98 \$ 5.00+0 \quad \mathbf{1 2 / 1 / 8 7 6 8 3}$ real circuit, such as the composition of synthetic materials, membrane oxygenators, and heparin coatings, have resulted in considerably less blood activation. Despite these improvements, however, excessive postoperative blood loss remains one of the major complications of cardiac operations. ${ }^{2}$ These bleeding disorders are thought to be caused at least in part by impairment of platelet function. Several investigators have demonstrated that the platelet-specific glycoprotein (GP) Ib, an important adhesion receptor, disappears from the platelet surface during $\mathrm{CPB}^{3} \mathrm{GPIb}$ is the receptor for von Willebrand factor, a protein that mediates the interaction between platelets and proteins such as collagen in the exposed subendothelium of a damaged vessel wall. ${ }^{4}$ A decreased expression of GPIb has therefore been suggested to lead to a decreased adhesive capacity and thus to result in prolonged bleeding and excessive blood loss. ${ }^{2,5}$ This hypothesis 
has been confirmed by the finding that administration of aprotinin, a bovine protein that acts as a nonselective inhibitor of serine proteases, prevented the disappearance of GPIb and simultaneously reduced the blood loss and the use of blood products during CPB. ${ }^{6}$ Several investigators could not demonstrate the disappearance of GPIb, however, and decreased expression of GPIb in systemic blood therefore remains under debate..$^{3,7-10}$

Boisclair and coworkers ${ }^{11}$ demonstrated that coagulation is initiated during CPB not only by the contact between blood and the extracorporeal circuit but also through a tissue factor-factor VII dependent pathway. More recently, direct evidence was provided that the expression of tissue factor is enhanced in the surgical wound and that the extrinsic coagulation cascade becomes activated. ${ }^{12}$ In the surgical wound, blood oozes from damaged vessels and makes extensive contact with the damaged vessel wall and underlying tissues. This blood accumulates in the pericardial cavity, from which it is removed by suction. Periodically the pericardial blood is returned into the patient. Thus, whereas previous studies suggested that blood is activated predominantly by contact activation, more recent studies indicate that the contribution of the material-independent pathway of blood activation-the operation itself-may be of much greater importance than previously thought. ${ }^{12,13}$ Our aim in this study was to establish whether the expression of GPIb is indeed reduced in patients undergoing coronary artery bypass grafting, whether this is associated with extensive general platelet activation, and whether these phenomena are present in both systemic and, perhaps more considerably, pericardial blood.

\section{Material and methods}

Clinical study. This study was approved by the Local Ethical Committee. After informed consent was obtained, seven patients undergoing elective coronary artery bypass grafting entered the study. No patient was older than 85 years or had evidence of severe heart failure, renal or hepatic dysfunction, or a bleeding diathesis. No patient was treated with coumarin derivatives, aspirin, dipyridamole, or nonsteroidal antiinflammatory agents within 5 days before the operation. The study patients did not receive antifibrinolytic agents or aprotinin during CPB.

CPB and anesthesia. Anesthesia was induced and maintained with weight-related doses of fentanyl, sufentanil, midazolam, or propofol and pancuronium. The extracorporeal circuit consisted of a Dideco D704 compact-flow system oxygenator and S3 roller pump (Stöckert, Munich, Germany). The priming of the extracorpo- real circuit contained lactated Ringer's solution $(1.3 \mathrm{~L})$, human albumin (200 ml 20\% weight/volume), mannitol (100 $\mathrm{ml} \mathrm{20 \%} \mathrm{weight/volume),} \mathrm{sodium} \mathrm{hydrogen} \mathrm{carbonate}$ (50 $\mathrm{ml} 8.4 \%$ weight/volume), heparin $(50 \mathrm{mg}$ ), and cefamandol $(2 \mathrm{~g})$. Each patient received dexamethasone (1 $\mathrm{mg} / \mathrm{kg}$ ). Volume was corrected with lactated Ringer's solution. Heparin $(3 \mathrm{mg} / \mathrm{kg})$ was given intravenously before cannulation of the aorta and repeated in a dose of 50 $\mathrm{mg}$ whenever the activated clotting time (Hemochron; International Technidyne Corp., Edison, N.J.) was shorter than 480 seconds. Pump flows ranged from 2.0 to 2.4 $\mathrm{L} / \mathrm{m}^{2} /$ min during hypothermia $\left(28^{\circ}\right.$ to $\left.32^{\circ} \mathrm{C}\right)$. Myocardial protection was achieved with modified St. Thomas' Hospital solution infused in the aortic root. After decannulation heparin was neutralized with protamine sulfate in a 1:1 ratio.

Blood acquisition. All systemic blood samples were drawn from the same central venous line. The blood samples from the pericardial cavity were taken directly from the cavity with a $10 \mathrm{ml}$ syringe. Sampling points were after induction, before skin incision (sample point 1), 5 minutes after the start of CPB (sample point 2), 10 minutes before release of the aortic crossclamp, at the start of the last distal anastomosis (sample point 3 ), and before protamine administration but after removal of the atrial canula (sample point 4). Systemic blood samples were taken at sampling points 1 through 4 and samples from the pericardial cavity at points 2 through 4 . Blood samples were taken simultaneously at each sampling point. The hematocrit of systemic blood decreased from $0.33 \pm 0.02 \mathrm{~L} / \mathrm{L}$ to $0.25 \pm 0.05 \mathrm{~L} / \mathrm{L}$ (sample point 2 ), $0.22 \pm 0.03 \mathrm{~L} / \mathrm{L}$ (sample point 3 ), and $0.23 \pm 0.02 \mathrm{~L} / \mathrm{L}$ (sample point 4 ). This decrease resulted from hemodilution with the priming fluid of the extracorporeal circuit. The hematocrit of pericardial blood samples decreased to $0.21 \pm 0.05 \mathrm{~L} / \mathrm{L}$ (sample point 2 ), $0.06 \pm 0.04 \mathrm{~L} / \mathrm{L}$ (sample point 3 ), and $0.20 \pm 0.03 \mathrm{~L} / \mathrm{L}$ (sample point 4 ). The low hematocrit of pericardial blood at sample point 3 was caused by hemodilution with saline and cardioplegic solutions. Although the platelet count closely reflected the changes in hematocrit, this count was not reliable in pericardial blood at sample point 3 because of the low platelet number and the presence of high concentrations of particles or cell fragments, which were erroneously counted as platelets. However, this artifactually increased platelet count did not influence the measurement of the antigen expression on the platelet surface because such measurements were performed only on the particles with GPIb expression (platelet material) and the proper forward and sideward light scatter characteristics of the cell population as assessed by flow cytometry (see the Flow Cytometric Analysis section).

Monoclonal antibodies. The following monoclonal antibodies were used in this study. Anti-GPIb (CLB-MB45) was obtained from the Central Laboratory of the Netherlands Red Cross Bloodtransfusion Service, Amsterdam, The Netherlands. The antibody is directed against GPIb- $\alpha$. Anti-GPIb was biotinylated as described by Hnatowich and colleagues. ${ }^{14}$ Fluorescein isothiocyanate (FITC)-labeled PAC-1 was provided by Dr. S. J. Shattil, University of Pennsylvania, Philadelphia. This antibody is directed against the fibrinogen-binding conformation of 
A

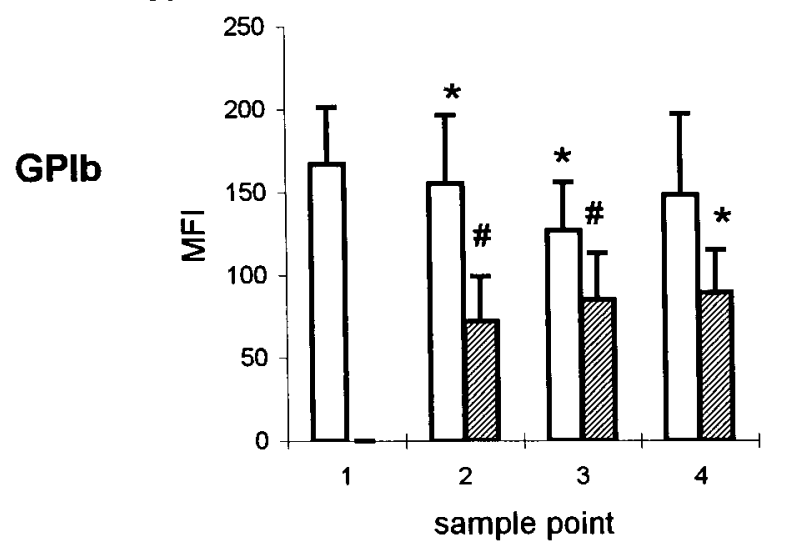

B

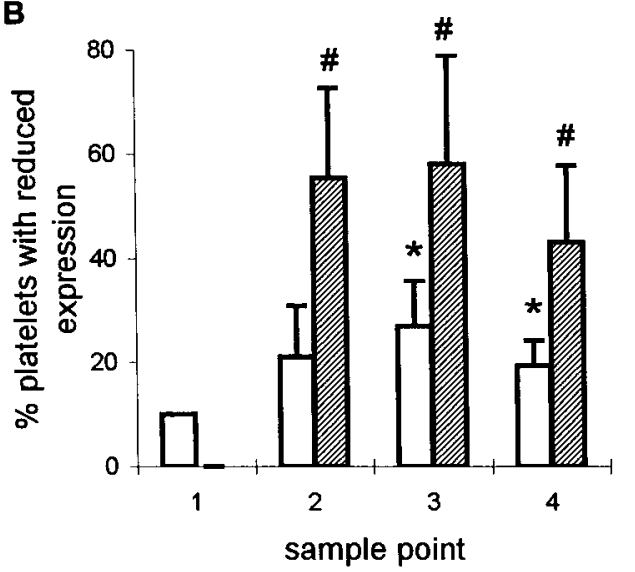

Fig. 1. Expression of GPIb in systemic blood (open bars) and pericardial blood (hatched bars) during CPB. A, MFI, as an indicator of the activation status of the total platelet population $(n=7$, mean \pm standard deviation). B, Percentage of platelets with decreased GPIb expression. The threshold for platelets with reduced expression was arbitrarily set at $10 \%$ for the first systemic blood sample $(n=7$, mean \pm standard deviation). Significant differences from sample point 1 are represented by asterisk $(p<0.05)$ and pound sign $(p<0.01)$.

the activated GPIIb-IIIa complex. ${ }^{15}$ FITC-labeled antiCD63 and FITC-labeled anti-CD62P were obtained from Immunotech, Marseilles, France. CD63 is present as a transmembrane protein of lysosomal membranes and is not found on resting platelets. On platelet activation, lysosomal membranes fuse with the cell membrane; concurrent with secretion, CD63 appears on the plasma membrane. CD62P, also called platelet activation-dependent granule-external membrane protein or P-selectin, is a transmembrane protein of $\alpha$-granules and is, like CD63, not present on resting platelets. Like CD63, it appears concurrently with secretion. Phycoerythrin-conjugated streptavidin was obtained from Dakopatts, Glostrup, Denmark.

Flow cytometric analysis. Double-label whole-blood flow cytometry was performed essentially as described by Abrams and colleagues ${ }^{15}$ and Shattil and associates ${ }^{16}$ with some modifications. Blood was collected in $0.32 \%$ trisodium citrate (final concentration). Within 5 minutes after sampling, $5 \mu \mathrm{l}$ aliquots of blood were added to tubes containing $30 \mu \mathrm{l} \mathrm{N}$-2-hydroxyethylpiperazine- $N$-2-ethanesulfonic acid (HEPES) buffer (137 mmol/L sodium chloride, $2.7 \mathrm{mmol} / \mathrm{L}$ potassium chloride, $1.0 \mathrm{mmol} / \mathrm{L}$ magnesium chloride, $5.6 \mathrm{mmol} / \mathrm{L}$ glucose, $20 \mathrm{mmol} / \mathrm{L}$ HEPES, 1 $\mathrm{mg} / \mathrm{ml}$ albumin, $3.3 \mathrm{mmol} / \mathrm{L}$ sodium phosphate, $\mathrm{pH}$ 7.4), 5 $\mu \mathrm{l}$ anti-GPIb $(5 \mu \mathrm{g} / \mathrm{ml}$ final concentration), and, where indicated, $5 \mu \mathrm{l}$ of a second FITC-labeled monoclonal antibody directed against CD62P, CD63, or the fibrinogen-binding conformation of the GPIIb-IIIa complex (monoclonal antibody PAC-1). After 15 minutes of incubation at room temperature in the dark, $5 \mu$ l tenfolddiluted phycoerythrin-conjugated streptavidin was added. After another 15 minutes of incubation at room temperature in the dark, $2.5 \mathrm{ml}$ HEPES buffer containing 0.2\% paraformaldehyde (weight/volume) was added. No changes in the expression of surface antigens occurred within 48 hours after fixation if platelets were prepared according to this protocol (Berckmans RJ, unpublished data). To verify our method of platelet activation analysis by expression of the activation markers, whole blood obtained from healthy volunteers $(n=8)$ and anticoagulated with $0.10 \mathrm{mmol} / \mathrm{L}$ sodium citrate (final concentration) was stimulated with $10 \mu \mathrm{mol} / \mathrm{L}$ adenosine diphosphate in vitro. The mean fluorescence intensity (MFI) for FITC-labeled anti-GPIb decreased from $218 \pm 20$ to $147 \pm 24$, MFI for FITC-labeled anti-CD62P increased from $3.6 \pm 0.5$ to $10.8 \pm 4.3$, MFI for FITC-labeled anti-CD63 increased from $5.6 \pm 0.8$ to $9.7 \pm 1.5$, and MFI for FITC-labeled PAC-1 increased from $11.3 \pm 4.2$ to $79 \pm 32$ (mean \pm standard deviation). These data indicate that the procedure used to label and fix the platelets can detect platelet activation by increased antigen expression of activation markers as well as reduced expression of GPIb.

The samples were analyzed in a FACScan flow cytometer with PC-lysis software (Becton Dickinson, San Jose, Calif.). Both forward light scatter and sideward light scatter were set at logarithmic gain, and platelets were found in whole blood by analyzing both phycoerythrinGPIb fluorescence at $585 \mathrm{~nm}$ and light-scattering profile. The surface expression of activation markers on a population of 5000 platelets was determined by the fluorescence intensity of the FITC label at $515 \mathrm{~nm}$. The threshold for platelet activation was set at $2 \%$ for CD62P and CD63 and 5\% for PAC-1 with the first systemic blood sample of each patient. The threshold for platelets with reduced expression of GPIb was arbitrarily set at $10 \%$. Surface expressions of CD62P, CD63, PAC-1, and GPIb were compared with those in the first systemic blood sample. Data are expressed as the percentage of platelets with decreased (GPIb) or increased (CD62P, CD63, PAC-1) expression with respect to these arbitrary thresholds. Thus 
Table I. Expression of CD62P, CD63, and PAC-1 in systemic and pericardial blood

\begin{tabular}{|c|c|c|c|c|c|c|c|}
\hline \multirow[b]{2}{*}{$M o A b$} & \multicolumn{4}{|c|}{ Systemic blood } & \multicolumn{3}{|c|}{ Pericardial blood } \\
\hline & 1 & 2 & 3 & 4 & 2 & 3 & 4 \\
\hline CD62P (MFI) & $5.4 \pm 2.7$ & $8.0 \pm 9.1$ & $6.1 \pm 5.5$ & $6.3 \pm 5.6$ & $4.2 \pm 1.1$ & $5.0 \pm 1.9$ & $7.4 \pm 6.0$ \\
\hline CD62P (\% act. plt.) & 2.0 & $4.4 \pm 4.4$ & $2.3 \pm 1.7$ & $1.7 \pm 1.6$ & $1.3 \pm 1.0$ & $3.4 \pm 4.7$ & $2.3 \pm 1.6$ \\
\hline CD63 (MFI) & $7.6 \pm 3.5$ & $8.7 \pm 6.5$ & $7.1 \pm 3.3$ & $8.2 \pm 6.6$ & $6.4 \pm 2.4$ & $7.7 \pm 4.2$ & $9.0 \pm 8.3$ \\
\hline CD63 (\% act. plt.) & 2.0 & $2.9 \pm 1.3$ & $2.1 \pm 0.9$ & $1.3 \pm 1.0$ & $1.6 \pm 0.8$ & $3.6 \pm 3.8$ & $2.6 \pm 2.1$ \\
\hline PAC-1 (MFI) & $12.7 \pm 9.1$ & $13.4 \pm 3.0$ & $8.7 \pm 2.7$ & $8.3 \pm 3.0$ & $11.9 \pm 4.6$ & $20.2 \pm 9.8$ & $8.7 \pm 4.3$ \\
\hline PAC-1 (\% act. plt.) & 5.0 & $9.2 \pm 1.9$ & $10.0 \pm 5.1$ & $4.0 \pm 1.4$ & $7.6 \pm 7.5$ & $8.0 \pm 4.7$ & $6.0 \pm 3.2$ \\
\hline
\end{tabular}

Data are presented as mean \pm standard deviation. $M o A B$, Monoclonal antibody; \% act. plt., \% activated platelets.

subpopulations of activated platelets may become apparent. ${ }^{17}$ The data are also expressed as MFI, as an indicator of the antigen expression of the total platelet population.

Statistical analysis. Comparisons within groups were assessed with the Wilcoxon signed-rank test and comparisons between groups were assessed with the MannWhitney U test with SPSS for Windows version 5.1 (SPSS, Inc., Chicago, Ill.).

\section{Results}

Clinical results. No complications occurred during or after the operation. The demographic data from the patients were as follows: six male patients and one female patient, mean age 65 years (range 47 to 72 years), mean CPB time 94 minutes (range 45 to 158 minutes), mean aortic crossclamp time 47 minutes (range 21 to 72 minutes), and mean postoperative blood loss within 24 hours after arrival at the intensive care unit $907 \mathrm{ml}$ (range 435 to $1865 \mathrm{ml}$ ).

Platelet activation analysis. Fig. $1(A)$ shows the expression of GPIb on the platelet surface, as assessed by the MFI, as a measure for the mean surface antigen density. In systemic blood the MFI of GPIb decreased by $10 \%$ at the start of CPB (sample point $2 ; p=0.03$ ), 30\% before release of the aortic crossclamp (sample point $3 ; p=0.04$ ), and $15 \%$ at the end of CPB (sample point $4, p=$ 0.35). The MFI of GPIb in pericardial blood at sample point 2 decreased by $50 \%$ versus (systemic) sample point $1(p=0.003), 51 \%$ before release of the aortic crossclamp (sample point $3 ; p=0.009$ ), and $41 \%$ at the end of CPB just before the start of protamine (sample point $4, p=0.05$ ).

To investigate the possibility that the observed decrease in GPIb expression was mainly caused by extensive activation of only a subpopulation of the platelets, the percentage of platelets with reduced expression of GPIb was measured. The threshold was arbitrarily set at $10 \%$ of the total population with the first systemic blood sample of each patient. All subsequent blood samples were compared with this first blood sample. Fig. 1 (B) shows that the number of platelets with reduced expression of $\mathrm{GPIb}$ in systemic blood increased from the arbitrary $10 \%$ (sample point 1 ) to $21 \%$ (sample point $2 ; p=$ 0.35 ), 27\% (sample point $3 ; p=0.02$ ) and $24 \%$ (sample point $4 ; p=0.04$ ). In pericardial blood the number of platelets with reduced expression of GPIb increased to $55 \%$ (sample point $2 ; p=0.001$ ), $58 \%$ (sample point $3 ; p=0.001$ ), and $43 \%$ (sample point $4 ; p=0.001$ ). These findings closely paralleled the observed decrease in expression of GPIb seen as MFI, indicating that the decreased MFI is a feature of the whole platelet population and not of a subpopulation.

In parallel, the expressions of the activation markers CD62P, CD63, and PAC-1 were studied (Table I). To our surprise, no increased expression was observed, not even in pericardial blood, in which we had expected platelets to be strongly activated.

\section{Discussion}

As in most other studies, we found a decrease in expression of GPIb in systemic blood (10\% to $30 \%$ ). In pericardial blood this expression decreased by approximately $50 \%$. It may seem surprising that we did not notice a subpopulation of platelets with a GPIb surface expression reduced by $50 \%$ in the systemic circulation as a result of return of the pericardial blood during the operation. During our study, however, which involved routine operations in patients at low risk, the amount of pericardial blood returned to the patient was approximately 0.5 L. Because pericardial blood is diluted about tenfold, this is comparable to approximately $50 \mathrm{ml}$ undiluted whole blood. When returned into the systemic circulation, the number of platelets that originated from pericardial blood would be $1 \%$ of the total platelet population or less, which is too low to be accurately detected by flow cytometry. Alternatively, the absence of such a subpopulation may 
be caused either by adsorption of platelets to the surface of the extracorporeal circuit or by clearance by the liver and the reticuloendothelial system. Conversely, such a small volume of platelets from the pericardial blood can not cause the reduced GPIb expression detected on the platelets in the systemic circulation. This would imply that the platelets in the circulation also become affected by nonpericardial processes. In more complicated operations, larger blood volumes are being collected in the pericardial cavity and returned into the patient; thus they may become more important for the overall systemic blood platelet activation analyses.

In this study we incubated the platelets in whole blood for 30 minutes at room temperature, with the antibodies added before fixation. Although this method optimizes the detection of all platelets present, it may lead to some underestimation of the GPIb disappearance. Michelson and colleagues ${ }^{18,19}$ reported that plasmin and thrombin at $37^{\circ} \mathrm{C}$ decrease GPIb expression as much as $30 \%$ to $50 \%$ and $20 \%$ to $50 \%$ of the initial value, respectively. After inactivation of the plasmin or thrombin, platelets can replenish their surface GPIb from an intraplatelet pool as much as $75 \%$ to $80 \%$ and $70 \%$ to $90 \%$ of the initial value, respectively. Still, we chose the method of antibody incubation before instead of after fixation because the latter method requires isolation of the fixed platelets before incubation with antibodies. This isolation may result in major loss of platelets, with a high risk of losing subpopulations. In addition, the fixative changes the epitopes and thus necessitates use of different antibodies. ${ }^{16}$

The mechanism behind the disappearance of GPIb remains to be established. For instance, whether proteolysis of GPIb occurs in whole blood is unknown, because the in vitro experiments from Michelson and colleagues ${ }^{18,19}$ were performed with high concentrations of plasmin and washed platelets, in the absence of plasmin inhibitors. Similar findings were reported for cathepsin $\mathrm{G}$, which reduced the expression of GPIb in a washed platelet system but not in whole blood. ${ }^{20}$ Compared with systemic blood, pericardial blood contains relatively high concentrations of the thrombin-antithrombin III complex, tissue plasminogen activator, and fibrin degradation products, indicating that especially in pericardial blood both thrombin and plasmin are generated despite anticoagulation with heparin. ${ }^{12,13,21}$ Whether thrombin, plasmin, or both are responsible for the disappearance of GPIb in pericardial blood is a subject for further investigation.
The clinical relevance of GPIb disappearance during $\mathrm{CPB}$ remains under debate. To determine the role of GPIb under flow conditions, we recently performed experiments with whole blood from healthy volunteers under flow conditions in which GPIb had been partially "knocked out" by a monoclonal antibody or by internalization after stimulation of the thrombin receptor. As much as $50 \%$ to $60 \%$ disappearance of GPIb did not affect platelet adhesion under flow conditions on various substrates, such as collagen type III and von Willebrand factor. ${ }^{22}$ These findings are in line with the absence of bleeding complications in patients heterozygous for Bernard-Soulier disease and indeed question the functional consequences of GPIb disappearance for platelet adherence during CPB in patients at low risk. On the other hand, because GPIb functions not only as a platelet adhesion receptor but also as a high-affinity receptor for thrombin, it is possible that a partial disappearance of GPIb affects platelet activation by thrombin. ${ }^{23,24}$

In this study no changes were found in expression of the activation markers CD62P, CD63, and PAC-1. The absence of an increase in expression of CD62P may be explained by the subsequent release of this receptor from the surface of activated platelets, as shown recently by Michelson and coworkers. $^{25}$ In addition, Santoso and associates ${ }^{26}$ described receptor capping on platelet stimulation in washed platelets as a result of incubation of unfixed platelets with specific monoclonal antibodies against GPIIb-IIIa. We cannot exclude the possibility that receptor capping also occurs in whole blood; however, it does not prevent the detection of platelet activation, as shown by the response of blood from healthy volunteers on stimulation (see Material and Methods). On the other hand, the binding of the monoclonal antibody PAC- 1 may be preceded by binding of fibrinogen, making this GPIIb-IIIa conformation inaccessible to PAC-1. At present we do not have an explanation for the absence of an increased CD63 expression, except by assuming that extensive platelet activation with the associated secretory reaction is evidently absent.

Taken together, the data indicate that the GPIb molecules disappear in part from the platelet surface in the systemic blood, and more notably in the pericardial blood, without signs of extensive platelet activation.

We thank Dr. P. G. M. Jansen, Department of Cardiac Surgery, University Hospital Vrije Universiteit, Amster- 
dam, The Netherlands, clinical epidemiologist and biostatistician, for his statistical advice.

\section{REFERENCES}

1. Edmunds LH Jr. Blood-surface interactions during cardiopulmonary bypass. J Card Surg 1993;8:404-10.

2. Woodman RC, Harker LA. Bleeding complications associated with cardiopulmonary bypass. Blood 1990;76:1680-97.

3. Kondo C, Tanaka K, Takagi K, Shimono T, Shinpo H, Yada I, et al. Platelet dysfunction during cardiopulmonary bypass surgery: with special reference to platelet membrane glycoproteins. ASAIO J 1993;39:M550-3.

4. Sixma JJ, van Zanten H, Banga JD, Nieuwenhuis HK, de Groot PG. Platelet adhesion. Semin Hematol 1995;32:89-98.

5. Tabuchi N, de Haan J, Boonstra PW, Huet RC, van Oeveren W. Aprotinin effect on platelet function and clotting during cardiopulmonary bypass. Eur J Cardiothorac Surg 1994;8:8790.

6. Bidstrup BP, Harrison J, Royston D, Taylor KM, Treasure T. Aprotinin therapy in cardiac operations: a report on use in 41 cardiac centers in the United Kingdom. Ann Thorac Surg 1993;55:971-6.

7. Rinder CS, Mathew JP, Rinder HM, Bonan J, Ault KA, Smith BR. Modulation of platelet surface adhesion receptors during cardiopulmonary bypass. Anesthesiology 1991;75:56370

8. Bertolino G, Locatelli A, Noris P, Maurelli M, Ceriana P, Mazzini G, et al. Platelet composition and function in patients undergoing cardiopulmonary bypass for heart surgery. Haematologica 1996;81:116-20.

9. Kestin AS, Valeri R, Khuri SF, Loscalzo J, Ellis PA, MacGregor $\mathrm{H}$, et al. The platelet function defect of cardiopulmonary bypass. Blood 1993;82:107-17.

10. Metzelaar MJ, Korteweg J, Sixma JJ, Nieuwenhuis HK. Comparison of platelet membrane markers for the detection of platelet activation in vitro and during platelet storage and cardiopulmonary bypass surgery. J Lab Clin Med 1993;121: 579-87.

11. Boisclair MD, Lane DA, Philippou H, Esnouf MP, Sheikh S, Hunt B, et al. Mechanisms of thrombin generation during surgery and cardiopulmonary bypass. Blood 1993;82:3350-7.

12. Chung JH, Gikakis N, Rao K, Drake TA, Colman RW, Edmunds LH Jr. Pericardial blood activates the extrinsic coagulation pathway during clinical cardiopulmonary bypass. Circulation 1996;93:2014-8.

13. de Haan J, Boonstra PW, Monnink SHJ, Ebels T, van Oeveren W. Retransfusion of suctioned blood during cardiopulmonary bypass impairs hemostasis. Ann Thorac Surg 1995;59:901-7.
14. Hnatowich J, Verzin F, Ruscowski M. Investigations of avidin and biotin for imaging applications. J Nucl Med 1987;28: 1294-302.

15. Abrams CS, Ellison N, Budzynski AZ, Shattil SJ. Direct detection of activated platelets and platelet-derived microparticles in humans. Blood 1990;75:128-38.

16. Shattil SJ, Cunningham M, Hoxie JA. Detection of activated platelets in whole blood using activation-dependent monoclonal antibodies and flow cytometry. Blood 1987;70:307-15.

17. Konijnenberg A, Stokkers EW, van der Post JAM, Schaap MCL, Boer K, Bleker OP, et al. Extensive platelet activation in preeclampsia compared with normal pregnancy: enhanced expression of cell adhesion molecules. Am J Obstet Gynecol 1997;176:461-9.

18. Michelson AD, Barnard MR. Plasmin-induced redistribution of platelet glycoprotein Ib. Blood 1990;76:2005-10.

19. Michelson AD, Benoit SE, Kroll MH, Li JM, Rohrer MJ, Kestin AS, et al. The activation-induced decrease in the platelet surface expression of the glycoprotein Ib-IX complex is reversible. Blood 1994;83:3562-73

20. Molino M, Di Lallo M, Martelli N, de Gaetano G, Cerletti C. Effects of leukocyte-derived cathepsin $\mathrm{G}$ on platelet membrane glycoprotein Ib-IX and IIb-IIIa complexes: a comparison with thrombin. Blood 1993;82:2442-51.

21. Tabuchi N, de Haan J, Boonstra PW, van Oeveren W. Activation of fibrinolysis in the pericardial cavity during cardiopulmonary bypass. J Thorac Cardiovasc Surg 1993;106: 828-33.

22. van Zanten GH, Heijnen HFG, Wu Y, Schut-Hese KM, Slootweg PJ, de Groot PG, et al. A 50\% reduction of platelet surface glycoprotein $\mathrm{Ib}$ does not affect platelet adhesion under flow conditions. Blood. In press 1998.

23. Greco NJ, Jamieson GA. High and moderate affinity pathways for alpha-thrombin-induced platelet activation. Proc Soc Exp Biol Med 1991;198:792-9.

24. Greco NJ, Tandon NN, Jones GD, Kornhauser R, Jackson B, Yamamoto N, et al. Contributions of glycoprotein $\mathrm{Ib}$ and the seven transmembrane domain receptor to increases in platelet cytoplasmic $\mathrm{Ca}^{2+}$ induced by alpha-thrombin. Biochemistry 1996;35:906-14.

25. Michelson AD, Barnard MR, Hechtman HB, MacGregor H, Connolly RJ, Loscalzo J, et al. In vivo tracking of platelets: circulating degranulated platelets rapidly lose surface Pselectin but continue to circulate and function. Proc Natl Acad Sci U S A 1996;93:11877-82.

26. Santoso S, Zimmermann U, Neppert J, Mueller-Eckhardt C. Receptor patching and capping of platelet membranes induced by monoclonal antibodies. Blood 1986;67:343-9. 